\title{
LENS
}

\section{A New Non-Linear Spectroscopy Facility}

Nestling below the villa on the Arcetri hill overlooking Florence where Galileo spent the last years of his life is a new building (see photo) that provides a collection of quite remarkable beams. In some respects it is only the scale of the centre which differentiates it from a normal beam factory: for the beams are of laser light and and the experiments conducted on table tops, albeit very carefully constructed.

The centre is the European Laboratory for Non-Linear Spectroscopy (LENS in Italian) established to provide state-of-the-art spectroscopic facilities to European researchers. Comprising four small laboratories specified according to the types of laser beams available (ultrafast, continuous wave, nanosecond and infrared), it responds to the need for a variety of facilities to exploit the vast range of opportunities opened up by the availability of pulsed and tunable high resolution laser sources.

These sources are used for non-linear and high resolution spectroscopy where new techniques can provide atomic and molecular information inaccessible by other methods. Indeed, LENS is one of the few facilities able to carry out a range of laser spectroscopies with high accuracy in both time and frequency, where costs are high and technical requirements stringent. Combining this with the means to produce supersonic streams of cold atoms and molecules with well defined characteristics provides exceptional possibilities.

Examples of recent results illustrate the precision which can be achieved. Fig. 1 indi-

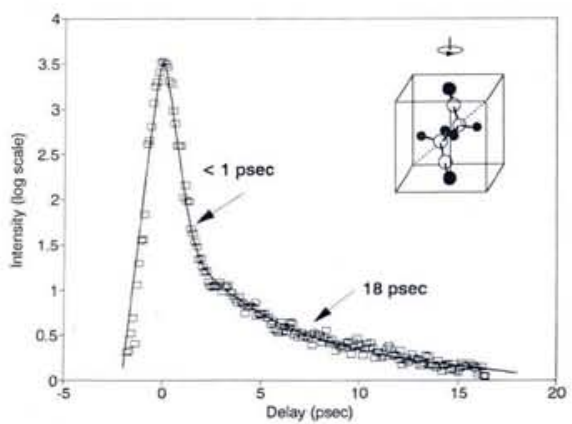

Fig. 1 - The relaxation kinetics of the plastic phase of succinonitrile measured by the sub-picosecond time resolved optical Kerr effect. Some polymers show a plastic phase characterized by the presence of translational order, as in real crystals, with orientational disorder. This disorder is dynamic and can be studied by light scattering in the frequency domain or directly in the time domain. The figure plots the transient optical Kerr effect intensity variation with time at $273 \mathrm{~K}$ for a typical decay and the insert represents the suggested mechanism for the slow (13 ps) component of the relaxation kinetics. cates that the relaxation of the plastic phase of a polymer (succinonitrile) occurs by a 13 ps rotation of the molecule, while Fig. 2 demonstrates that LENS's tunable far infrared spectrometer is able to resolve all of the rotational components of ozone in the sub-mm region (they are not resolved at high frequencies using conventional high resolution Fourier spectroscopy).

\section{A Quick Start}

The idea of a European spectroscopy laboratory gelled some 4-5 years ago at the University of Florence from a proposal by Professor Califano, a chemical physicist, in collaboration with Professor Arecchi, a renowned expert in quantum optics. Professor Califano, who was later joined by M. Inguscio, Professor of Optics in the Physics Department, became the Chairman of the Committee charged with creating the Laboratory. He will most likely be appointed LENS's Director once the formal structure is voted on this month by the Laboratory's Council of nine representatives drawn from participating institutions, including universities in Paris, Madrid and Florence. LENS will then be an independent research centre governed by its own statutes that passed into law by Act of Parliament on 30 July. Legal recognition will facilitate arrangements as it allows an operating budget to be established and permanent staff appointed some two years after the present building was completed and equipment installed, largely with the help of a financial support over a similar period from the Ministry of Education (responsibility has passed to the newly formed Ministry of Universities and Research and Technology created in April as part of a restructuring of Italian science).

The present buildings are, however, temporary as it is planned to move LENS to a new site at Sestro outside Florence where several CNR laboratories, the National Institute for Optics and Florence University's science faculty will be consolidated.

\section{Projects}

The European Community is seen as an important source of financial support, in addition to a roughly 1 MECU operating budget from the Ministry, but so far only one application has been approved. This is a LENS participation in the 6 MECU ISORAC collaboration to investigate atmospheric ozone as part of the EC's environmental programme STEP. A grant of about 2 MECU over two years from the EC's Large-Scale Facilities programme will be forthcoming in the next round of allocations to provide for access by users. There is also participation in the current EC SCIENCE Plan in a study of atomic hydrogen with Paris, and in the transfer of a mobile cluster ion source from Germany for use at LENS for several years to investigate metallic clusters. Postgra-

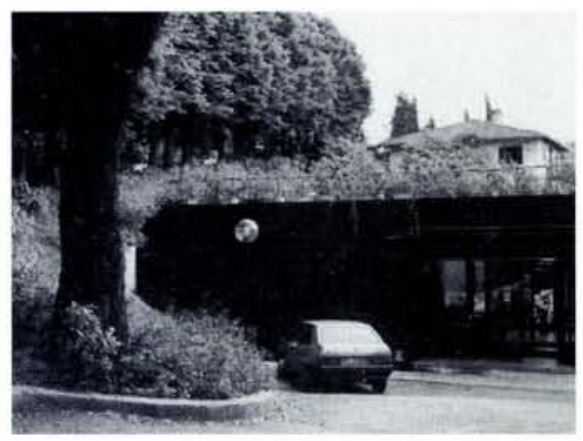

The LENS main building.

duates and scientists will be encouraged to apply through the centre for grants for $1-2$ month and $1-2$ year stays under the new EC human resources mobility scheme once the programme is launched (probably next year, see Europhysics News 22 (1991) 130). Meanwhile, proposals to other agencies are being pushed vigorously and further industrial contracts for service work sought.

The start-up phase involving 18 visiting scientists and 20 publications over 12 months demonstrated the centres importance. Facilities are available to participating institutions without overhead charge whereas visitors from other institutions are charged according to the instrument and time required. LENS will also act continue to act as a centre for meetings and courses.

All-in-all, LENS with its 10 or so individual lasers sources and the ancillary items representing a $6 \mathrm{MECU}$ investment in very specialised equipment is clearly set to provide an important focus for non-linear spectroscopy in the future.

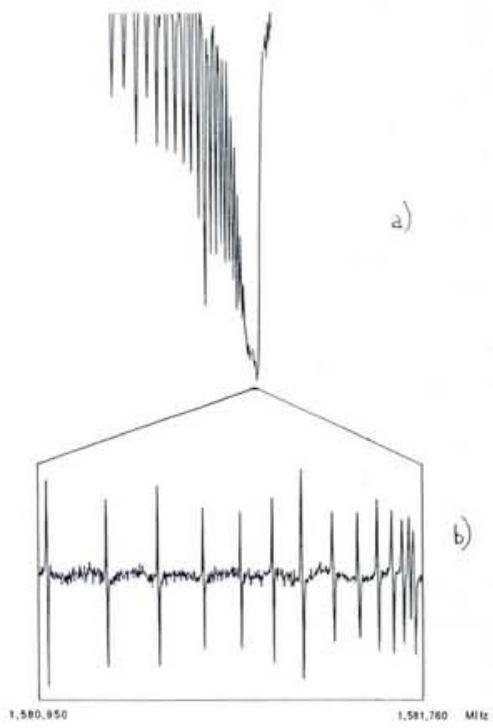

Fig. 2 - The pure rotational spectrum of ozone in the sub-mm range. a, upper) The spectrum taken with a high resolution $\left(0.003 \mathrm{~cm}^{-1}\right)$ Fourier transform spectrometer. $b$, lower) A portion of the spectrum obtained with the tunable far-infrared spectrometer at LENS. (spectral coverage: 0.3-6 $\mathrm{THz}$; instrumental linewidth and absolute accuracy: $50 \mathrm{~Hz}$ or $>10^{-7}$ ): all the components are resolved within the Doppler width. 